PRAXIS

ecucativa

Universidad Nacional de La Pampa

Facultad de Ciencias Humanas

Instituto de Ciencias de la Educación

para la investigación interdisciplinaria

\section{† 国埐 ICEN \\ Instituto de Ciencias de la Educación

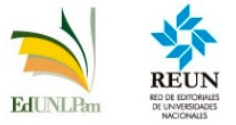

ISSN 2313-934X

SANTA ROSA, LA PAMPA, ARGENTINA

Correo electrónico: iceii@humanas.unlpam.edu.ar

Disponible en https://cerac.unlpam.edu.ar/index.php/praxis

Cambian los tiempos, las instituciones, los sujetos: reflexiones sobre la significatividad de las experiencias de aprendizaje en escuelas secundarias bonaerenses. Artículo de Analía Errobidart y Ana M. Viscaino. Praxis educativa, Vol. 26, No 1 enero - abril 2022. E - ISSN 2313-934X. pp. 1-19. https://dx.doi.org/10.19137/praxiseducativa-2022-260111

Esta obra se publica bajo Licencia Creative Commons 4.0 Internacional CC BY- NC-SA Atribución, No Comercial, Compartir igual

\title{
Cambian los tiempos, las instituciones, los sujetos: reflexiones sobre la significatividad de las experiencias de aprendizaje en escuelas secundarias bonaerenses
}

Times, institutions, subjects change: reflections on the significance of learning experiences

in Buenos Aires secondary schools

Tempos, instituições, mudanças de assunto: reflexões sobre o significado das experiências de aprendizagem nas escolas secundárias da província de Buenos Aires

\section{Analía Errobidart}

Universidad Nacional del Centro de la Provincia de Buenos Aires, Argentina analiaerrobidart@gmail.com

ORCID 0000-0002-6640-2462

\section{Ana M. Viscaino}

Universidad Nacional del Centro de la Provincia de Buenos Aires, Argentina

anaviscaino61@gmail.com

ORCID 0000-0003-4819-8522

Recibido: 2021-09-28 | Revisado: 2021-11-12 | Aceptado: 2021-12-07 


\section{Resumen}

El propósito de este artículo es reflexionar sobre la significatividad de las experiencias de aprendizaje escolares desde la perspectiva de un grupo de jóvenes estudiantes de escuelas secundarias de una localidad de la provincia de Buenos Aires. La reflexión y análisis se enmarca en resultados de investigación obtenidos de indagaciones realizadas antes y durante la pandemia de Covid-19. Las mismas pretenden atender a las condiciones de producción de los aprendizajes escolares que son identificadas, por la población estudiantil, como promotoras de significatividad. Sus voces se constituyen en datos relevantes para problematizar el encuentro pedagógico en los tiempos contemporáneos poniendo el acento en la dimensión intersubjetiva y cultural que dan sentido a la experiencia escolar.

Palabras clave: escuela secundaria; cambios; jóvenes; experiencias; significatividad

\section{Abstract}

The purpose of this article is to reflect on the significance of school learning experiences from the perspective of a group of young secondary school students from a town in the province of Buenos Aires. The reflection and analysis is part of the research results obtained from inquiries carried out before and during the Covid-19 pandemic. They seek to address the production conditions of school learning that are identified by the student population as promoters of significance. Their voices become relevant data to problematize the pedagogical encounter in contemporary times, emphasizing the intersubjective and cultural dimension that give meaning to the school experience.

Key-words: secondary school; changes; youths; experiences; significance

\section{Resumo}

O objetivo deste artigo é refletir sobre o significado das experiências de aprendizagem escolar a partir da perspectiva de um grupo de jovens estudantes do ensino médio de um município da província de Buenos Aires. A reflexão e análise fazem parte dos resultados da pesquisa obtidos em inquéritos realizados antes e durante a pandemia Covid-19. Procuram-se abordar as condições de produção da aprendizagem escolar que são apontadas pela população estudantil como promotoras de significados. Suas vozes constituem dados relevantes para problematizar o encontro pedagógico na contemporaneidade, enfatizando a dimensão intersubjetiva e cultural que dá sentido à experiência escolar.

Palavras-chave: ensino médio; mudanças; jovens; experiências; significado. 


\section{Introducción}

El propósito de este artículo es reflexionar sobre la significatividad de los aprendizajes escolares desde la perspectiva de un grupo de jóvenes estudiantes de escuelas secundarias de una localidad de la provincia de Buenos Aires. Estas reflexiones se sustentan en investigaciones realizadas en cinco escuelas secundarias bonaerenses durante los períodos 2017-2019 y 20192021, por integrantes del Núcleo de Actividades Científicas y Tecnológicas "Investigaciones en Formación Inicial y Prácticas Educativas" (NACT IFIPrac_Ed) de la Universidad Nacional del Centro de la Provincia de Buenos Aires. En tal sentido, las indagaciones se corresponden con períodos claramente diferenciales en torno de las condiciones en las que se han producido los aprendizajes escolares en cada caso y sobre los que se pretende producir el presente análisis. Los aprendizajes escolares y, en particular, aquellos que acontecen en el nivel de educación secundaria se ven profundamente tensionados ante las transformaciones sociales, culturales, científicas y tecnológicas que ha impuesto el ingreso al mundo globalizado. La intención de ampliar y democratizar el acceso a la educación formal del conjunto de la población juvenil, promovida por la Ley de Educación Nacional a partir del año 2006, se constituye, sin duda, en otro de los aspectos para tener en cuenta en este clima de tensiones, al igual que en los modos diferenciales de acceso promovidos por las escuelas particulares y los grupos, que dentro de cada una se conforman, para significar la tarea de enseñar y aprender. Es en este marco que se produce la indagación llevada adelante sobre los procesos de significatividad del aprender en el primer período de investigación mencionado. En tanto, en el segundo período, el proceso de investigación y de producción de la experiencia escolar se desarrolla en un contexto atravesado por las condiciones de gran conmoción que tiene lugar con la pandemia provocada por el COVID19.

La pandemia que se inició en Wuham, a fines de 2019, trajo nuevas problemáticas educativas al continente latinoamericano profundizando las desigualdades preexistentes y también el desinterés de muchas y muchos jóvenes por las propuestas educativas bajo la modalidad virtual y a distancia. Abordando las diferentes problemáticas que la pandemia aporta a la crisis de la educación secundaria, se considera que analizar, contextualizar y comprender los aspectos que hacen de una situación de enseñanza y aprendizaje un acto de significatividad aportará a la construcción de sentidos y contribuirá a suturar el desfasaje entre las propuestas educativas (virtuales o presenciales físicas) y los intereses de las y los jóvenes.

La crisis de la educación que atraviesa la Argentina y gran parte de los países de la región se nutre tanto de los contenidos seleccionados para ser enseñados y aprendidos en cada nivel como en las formas de enseñar en contextos escolares creados bajo la mirada del siglo XIX y con normas, reglas y pautas de funcionamiento del mismo siglo. Los elementos mencionados han sido tematizados y problematizados por autores del campo educativo que han recorrido la problemática de la crisis de la escolaridad, como Pérez Gómez (2017), Grimson y Tenti Fanfani (2015), Gimeno Sacristán (2013), Tenti Fanfani (2007), Tiramonti (2011), entre muchos/as otros/as. 
La emergencia educativa vivida en 2020 puso aún más de manifiesto que los contenidos escolares están desfasados de la realidad, carecen de utilidad y de sentido en relación con el mundo complejo que estamos viviendo, no solo vinculado al mundo del trabajo, sino a las capacidades que requiere un mundo dinámico como el actual. La escuela, el currículum y los métodos de enseñanza resultan obsoletos en relación con el acceso a diferentes y múltiples fuentes de información que los y las jóvenes tienen a su alcance y que es necesario aprender a procesar, tanto para poder interpretar el mundo cercano como el mundo global del que somos parte.

En Latinoamérica, a esos problemas persistentes en la historia educativa se le suman nuevas tensiones, más recientes, como las vinculadas con la extensión de la escolaridad formal de las y los jóvenes a la que hicimos referencia con anterioridad. En estos países con historia de desigualdad y exclusión, la extensión de la obligatoriedad de la educación hasta alcanzar el nivel secundario completo trajo aparejado el desafío de la inclusión educativa, primer factor fundamental para asegurar posteriores acciones educativas. En él, la tarea política del Estado y la acción cotidiana de las y los docentes han sido temas de diversos estudios.

En este artículo, recurriremos a datos construidos a lo largo de dos proyectos de investigación sobre escuelas secundarias y las actividades realizadas en el marco de ambos trabajos de indagación en el campo de estudios para identificar cómo los y las jóvenes valoran y significan los aprendizajes realizados en el tránsito por la escolaridad secundaria. La investigación se realizó en una ciudad intermedia del centro de la provincia de Buenos Aires. La perspectiva metodológica adoptada es de corte socioantropológico. Este tipo de investigación se define por ciertas características como: ser una investigación de corte cualitativo que se orienta a relevar procesos de interpretación; realizarse sobre la base de trabajo de campo y el registro en cuadernos de campo, un proceso posterior de distanciamiento del investigador en la elaboración de sus informes y el trabajo con un diseño flexible (Errobidart, 2021).

Se trata de los proyectos "La educación secundaria bajo el mandato de inclusión social. Sujetos, instituciones y prácticas" correspondientes al período 2017-2019 y al proyecto de investigación "Escuelas secundarias, configuraciones culturales y aprendizajes significativos" desarrollado en el período 2019-2021. Este último tuvo como uno de sus propósitos relevar información acerca de cómo los sujetos partícipes de diversas configuraciones sociales y culturales se vinculan con la educación secundaria obligatoria y cuáles son los aprendizajes que valoran. Este propósito se vio complejizado y resignificado a partir de las nuevas coordenadas que atravesaron la vida escolar en tiempos de pandemia.

La decisión de trabajar con ambos registros temporales se basa en la posibilidad de ubicar experiencias significativas en períodos previos a la pandemia del COVID-19 y otros que se producen en el contexto de aislamiento social, preventivo y obligatorio (denominado ASPO) durante el año 2020. El campo estuvo conformado por las mismas escuelas secundarias en ambos proyectos y los y las informantes (estudiantes, para este caso de estudio) fueron los/as 
mismos/as o variaron según la edad y condición de regularidad en la trayectoria formal de estudio.

El artículo consta, además de esta introducción, con un apartado que aborda las perspectivas conceptuales que, en el transcurso del siglo XX, y desde diferentes enfoques, dieron lugar a un análisis en torno de los procesos de significatividad en los procesos de aprender $y$ conocer. En un apartado subsiguiente, se desarrollan cuestiones metodológicas específicas del proyecto de investigación en el que se producen los hallazgos que problematizamos.

A continuación, se presentan las significaciones y valoraciones que se realizan a partir de las expresiones de las y los jóvenes estudiantes participantes de los estudios y, finalmente, realizamos algunas reflexiones que buscan interpretar la significatividad de los aprendizajes contenidas en encuentros pedagógicos que fueron especialmente valorados por los y las estudiantes a quienes consultamos.

\section{La significatividad del aprender y conocer en la Psicología del siglo XX}

La consideración de la significatividad en los procesos de aprendizaje no siempre fue un tema abordado por las corrientes de pensamiento psicológico desde los tiempos de la constitución de la Psicología como disciplina científica. Más bien, junto con la pregunta por la conciencia, se ha constituido en uno de los pilares de las discusiones epistemológicas que atravesaron este campo de estudio desde finales del siglo XIX y que, podríamos decir, aún hoy se sostienen, aunque de formas algo más solapadas.

La pregunta por el significado fue acompañada, en gran medida, de las inquietudes por ocuparse de la concienciai que caracterizó a algunos de los desarrollos teóricos de la psicología del aprendizaje que, sustentados en un enfoque epistemológico de corte constructivista, produjeron fuertes controversias y discusiones hacia fines del siglo XIX y principios del siglo XX. Ese fue un tiempo en el que las mayores tensiones a la hora de investigar y explicar la conducta y el desarrollo mental humano se instalaban en posiciones dicotómicas entre mundo interno y mundo externo, lo físico y lo mental, lo animal y lo humano o lo medible y cuantificable por sobre lo que forma parte de un mero acto de especulación destinado al campo de la filosofía.

Los desarrollos científicos del momento, regidos centralmente por el avance de la ciencia positivista, no veían con buenos ojos tomar nota de procesos que no podían ser medibles, cuantificables, es decir, objetivados a través de métodos de control empírico. Así, la conducta se tornó objeto de estudio privilegiado para las corrientes que adoptaron una perspectiva asociacionista. Tales posiciones se vieron acompañadas por estudios que, por el contrario, y buscando sentidos más ocultos de las manifestaciones conductuales, ingresaron a un debate que comienza a desplegarse con fuerza en el siglo XX, de la mano de teorías como la Gestalt, Psicogenética y Sociohistórica. Teorías que, en algunos casos, son recuperadas, en la década del 60 , en el contexto norteamericano en un tiempo regido por la desvitalización del Conductismo y la necesidad de comenzar a pensar los procesos culturales y de producción de significados en la cognición humana. 
A los fines del presente escrito, nos referiremos a estos movimientos de la psicología que, buscando encontrar algunas respuestas a la pregunta de la didáctica acerca de la diversidad de aprendizajes producidos en la incipiente escolaridad, se abocaron a comprender sentidos y procesos dinámicos y contextuados de construcción de conocimientos.

En particular, nos referimos a los estudios que dan especial relevancia a la producción de significados como perspectiva analítica del aprendizaje y el desarrollo humano, como es el caso de los estudios de Vygotski (2000) y Bruner (1998), y a la noción de aprendizaje significativo tal como la plantean Ausubel (1973) y Ausubel et al. (1991).

Vygostki (1978), en particular, ha sido uno de los psicólogos que, con mayor fuerza, sostiene, en este momento histórico, la necesidad de dar cuenta de la especificidad del desarrollo humano, de la constitución de aquellos procesos psicológicos superiores que nos diferencian del resto de los animales. Es en esta ardua empresa de la época que logra instalar una perspectiva sociocultural de los orígenes de los procesos mentales complejos, tal como el lenguaje y el pensamiento. La reconstrucción interna de una operación externa a la que Vygotski denomina internalización representa el mecanismo de cambio y transformación del desarrollo humano de la mano de la producción de significados que se abre en/desde la acción mediada que tiene lugar en el contexto cultural.ii

La naturaleza sociocultural e intersubjetiva del aprendizaje y desarrollo humano y los procesos de internalización implicados en ellos se torna en una idea fértil para la producción de derivaciones hacia el ámbito de la educación formal que adquieren especial forma a través de la noción de Zona de Desarrollo Próximo. Noción que identifica al desarrollo como un proceso progresivo, dependiente del aprendizaje y del mecanismo de internalización, que irá demarcando momentos y zonas de avance y consolidación a través de niveles de desarrollo real o potencial de los niños y niñas. Este modo de pensar las relaciones entre aprendizaje y desarrollo permite redimensionar el lugar del educador en las prácticas de instrucción en tanto actor significativo en la promoción de avances en los aprendizajes de los/as estudiantes y, en consecuencia, en su desarrollo.

En línea con esta perspectiva teórica, Bruner (1991), en una segunda etapa de su producción teórica, comienza a dedicar especial atención al lenguaje y la narración como producto de fuerzas históricas y culturales y a la producción de significados como una actividad cultural que organiza la visión de las personas acerca de sí mismos, los demás y el mundo en el que viven. Una psicología popular, al decir del autor, que permite reflexionar sobre la naturaleza, causas y consecuencias de los estados intencionales del ser humano y, de esta manera, buscar los fundamentos explicativos de lo que ha dado en llamar una "acción situada" (1991, p. 28-34).

Estas construcciones, en consonancia con lo planteado por Vygotski, fueron pensadas como parte de un entramado de significaciones que se encuentran distribuidas en el espacio interpersonal y arraigados en la historia común. De este modo, las explicaciones acerca de la condición humana no tendrán sentido y plausibilidad si no son "interpretadas a la luz del mundo simbólico que constituye la cultura humana" (Bruner, 1991, p. 132-133). 
Una psicología que se ocupe esencialmente del significado, tema que resulta olvidado en la línea teórica denominada Procesamiento de la Información, como plantea Bruner, se convertirá inevitablemente en una Psicología cultural. Es en el contexto de la participación del ser humano en la cultura que el significado se torna público y compartido. Es decir, "vivimos públicamente mediante significados públicos y mediante procedimientos de interpretación y negociación compartidos. La interpretación, por 'densa' que llegue a ser, debe ser públicamente accesible, o la cultura caerá en la desorganización y sus miembros individuales con ella" (Bruner, 1991, p. 29).

Esta perspectiva cultural para pensar la producción de significados que pueblan la mente humana resulta relevante al tema que nos ocupa en este escrito, en tanto permite redimensionar la dinámica de producción significante como parte de un entramado que se configura social y personalmente, llevando a los sujetos a valerse de los significados de la cultura en la que viven al tiempo que lograr modos posibles de implicarse subjetivamente en ella.

Hasta aquí, buscamos dar cuenta de algunos de los desarrollos teóricos de la psicología que han insistido en mostrar la impronta cultural en la constitución y desarrollo de los procesos psicológicos superiores involucrados en el aprendizaje y desarrollo específicamente humano.

Ahora bien, el abordaje de la noción de significado, en el marco de un proceso educativo escolarizado, es desarrollada por Ausubel (1973) quien, desde una perspectiva cognitiva en la producción de conocimiento escolar, pretende atender a una Psicología Educativa que dé marco a la formación del profesorado. Esta perspectiva teórica nos presenta el doble desafío de problematizar, por un lado, y en un sentido epistemológico, la entidad del conocimiento y la investigación educativa acerca del aprender escolar. Por otro, la implicancia de los/as profesores/as en la elección o creación de los métodos de enseñanza más eficaces para favorecer el aprendizaje del salón de clases en sus condiciones complejas y cambiantes de producción.

Se abre aquí la pregunta por la naturaleza del aprendizaje escolar, los factores sociales, cognoscitivos y afectivos que lo influyen, así como las condiciones y sentidos que lo producen (Ausubel et al., 1991). La especificidad del aprendizaje escolar tiene, bajo esta perspectiva teórica, la particularidad de requerir un proceso de asimilación de conceptos científicos producto de una actividad intencionada de instrucción que no tiene por qué ser memorístico, sino que debería promover su comprensión. En esta perspectiva teórica, el proceso de comprensión estará dado por un proceso de reorganización en la estructura de conocimiento de quien aprende en tanto tenga lugar una doble significatividad. Por un lado, una significatividad vinculada a la organización y presentación del contenido en tanto debe contemplar una estructuración lógica desde la cual sus elementos constitutivos sean organizados de modo significativo, no arbitrario o yuxtapuesto. Por otro lado, una significatividad psicológica que requiere no solo de la motivación y disposición del/de la estudiante a establecer relaciones significativas, sino también a que el nuevo contenido pueda relacionarse, de modo "no arbitrario y sustancial (no al pie de la letra) con lo que el alumno ya sabe", es decir, que pueda relacionarse significativamente con la estructura cognitiva de la que dispone (Ausubel et al., 1991). 
Pensamos que la elección de estos referentes teóricos de la psicología adopta un sentido particular para el análisis que aquí proponemos realizar en tanto nos permite adentrarnos en una mirada que podría poner en diálogo la cultura y la cognición para pensar la significatividad del aprendizaje escolar. Dimensiones identificadas como relevantes de recuperar, problematizar y complejizar a través de los datos ofrecidos por las voces de los y las estudiantes con quienes dialogamos en ambos procesos de investigación.

\section{Perspectivas y dispositivos de investigación para relevar experiencias significativas de aprendizaje}

En este apartado, se hará referencia al contexto de las investigaciones desde las racionalidades que le dan origen (Giroux, 1992). Comprender la racionalidad subyacente nos remite a trabajos e informes anteriores (Chapato y Errobidart, 2011; Errobidart, 2016), donde la pregunta por los sentidos de educar ha cambiado, a la par del declive del proyecto moderno. Partimos de hacer nuestras las ideas expresadas por Lewkowicz (2004) acerca de que las transformaciones del Estado-Nación afectarían a las instituciones disciplinarias del Estado moderno y, entre ellas, a la escuela. En el caso de la escuela secundaria, además, la extensión progresiva de la obligatoriedad de la educación hasta completar todo el ciclo transformó drásticamente la lógica con que fue desarrollada desde su creación. A partir del ingreso a un nuevo modelo político, económico, social y cultural, las instituciones y los sujetos (en este caso, estudiantes, profesores/as, equipos de gestión escolar) sufren la destitución de sus funciones originarias propias de los Estados modernos e ingresan a conformar un "clima de anomia que impide la producción de algún tipo de ordenamiento" (Lewcowikz, 2004, p. 31).

En correspondencia con lo anterior, el trabajo de Francois Dubet $(2010,2011)$ muestra cómo la universalización de la educación se produce en contextos de declive del programa institucional que organizó y fortaleció el Estado moderno. Se trató de un programa donde las organizaciones estatales aseguraban homogeneización y trayectorias de socialización más o menos estables y el rol de la educación pública -en tanto conglomerado complejo de organizaciones estatales, como también de cada una de los establecimientos educativosaparecía como estable y comprensible para todos los sujetos que en ella se desenvolvían.

La universalización de la educación secundaria demanda que aquellos/as jóvenes que no transitaban por los establecimientos escolares se encuentren hoy frente a la obligación de hacerlo, sin necesariamente haber construido antes un sentido propositivo sobre el por qué y cómo de la obligatoriedad de la educación secundaria. Bajo estas condiciones, los/as jóvenes discuten, tensionan y resignifican el sentido asociado a la educación media y obligan a participar, en este proceso de reasignación de sentido, al resto de los actores escolares. En estas condiciones, docentes y directivos se encuentran ante situaciones cotidianas en las cuales su rol se ve interpelado y necesita ser explicado, justificado y volver a reconstituirse como un actor social competente y valorado para el ejercicio de la tarea educativa. 
En consecuencia, hoy, los establecimientos educativos son organizaciones con disputas, tensiones y negociaciones cotidianas en torno al sentido de la educación que suele provocar escenarios conflictivos que reclaman de la reconfiguración de los roles e identidades educativas modernas. Desde esta trama relacional, en el territorio (físico o virtual), la investigación se interroga por los sentidos que los actores institucionales construyen acerca de la escolaridad secundaria obligatoria y, en particular, sobre la significatividad de los aprendizajes que allí se posibilitan.

La pregunta por los sentidos de la educación secundaria que se construyen en los actuales escenarios escolares abrió un espectro de posibilidades mayor que el que nosotras habíamos anticipado. El campo de indagación se conformó por una muestra intencional de cinco escuelas secundarias: una escuela de gestión privada, con más de cien años de trayectoria; una escuela de gestión estatal con más de cien años de trayectoria -creada como escuela nacional y provincializada en los años 90-; una escuela dependiente de una universidad regional; una escuela secundaria de gestión estatal, rural; y una escuela de gestión estatal creada en 2008 a partir de la Ley de Educción Nacional (LEN) N²6.206/06.

Se relevaron diversos (y a veces contradictorios) sentidos que se construyen entre los sujetos-actores de este nivel educativo, sobre la educación secundaria (Errobidart, 2014; Errobidart, 2016); Viscaino, 2018). A partir de las investigaciones mencionadas, se pretendió profundizar en los sentidos construidos por ellos/as acerca de la significatividad que otorgaban a los aprendizajes transitados en cada contexto de indagación.

\section{Acerca del trabajo de campo}

El trabajo de campo en ambos proyectos de investigación, cuyos datos se presentan y discuten en este artículo, posibilitaron la visibilización de una realidad hasta entonces desconocida para nosotras, que se desarrolla día a día en las aulas, los pasillos, los patios y las esquinas de las escuelas secundarias de una ciudad intermedia ubicada al centro de la provincia de Buenos Aires, Argentina.

Los avances en el trabajo mostraron que la destitución de la capacidad instituyente de la escuela era explícita, pero, sin embargo, los y las jóvenes siguen concurriendo a ella. Es allí donde comenzamos a ampliar nuestra pregunta: si la escuela ya no puede instituir los valores que le dieron origen, si la escuela no puede enseñar los contenidos curriculares y su normatividad, es rebatida por las pautas de acción que cada cultura juvenil impregna en las diferentes instituciones, entonces, ¿qué sentidos de educar se producen en la escuela?

Una de las líneas de trabajo implementadas en el primer proyecto de investigación tuvo como objeto relevar las experiencias de aprendizaje más significativo logradas por las y los jóvenes en su paso por la escolaridad secundaria, es decir, aquellas que les condujeran a hablar de los sentidos que, para ellos/as, tienen las experiencias educativas. Consistió en la realización de talleres, entrevistas y narración de experiencias con estudiantes de sexto año de las escuelas seleccionadas. 
Los talleres tuvieron una duración de dos horas cada uno y se realizaron durante tres encuentros en cada escuela.

\section{Tabla 1}

Cantidad de estudiantes por escuelas que participaron de los talleres

\begin{tabular}{|l|l|l|}
\hline Escuela & Numero de divisiones & Cantidad de estudiantes \\
\hline Colegio Confesional (privado) & 1 & 29 \\
\hline Escuela preuniversitaria & 2 & \\
\hline Ex escuela nacional & 5 & 64 \\
\hline Escuela secundaria rural & pluriaño & 158 \\
\hline Escuela de reciente ceración & 1 & 7 \\
\hline Total: & 10 & 11 \\
\hline
\end{tabular}

Como cierre del dispositivo, se realizó una reunión plenaria de todos los/as estudiantes participantes de los talleres, en una modalidad interinstitucional. En el primer encuentro intrainstitucional con el grupo de investigadoras, los y las jóvenes definían lo que era una experiencia significativa de aprendizaje. Sobre esa hipótesis colectiva inicial, se trabajaron dos talleres posteriores, profundizando en el sentido de las experiencias seleccionadas por los y las jóvenes en cada uno de los cursos participantes (diez cursos en total). Corresponden a ese trabajo las expresiones que se analizan más adelante.

En el segundo proyecto, el trabajo de campo debió realizarse bajo la vigencia del aislamiento social. El análisis que se presentará se deriva de entrevistas realizadas con 30 estudiantes de quinto y sexto año de las escuelas secundarias vinculadas al núcleo de investigación que se ha tomado como referencia.

El guion de la entrevista fue común a las y los estudiantes, pero quedó a su elección el medio que se utilizaría para realizarla. El más elegido fue WhatsApp, aunque algunos accedieron a usar la plataforma Zoom.

\section{Relevamientos en el campo de la investigación en los dos períodos seleccionados}

La indagación por el sentido de experiencias significativas de aprendizaje que aquí proponemos se nutre de las voces de estudiantes de nivel secundario que formaron parte de procesos de investigación mencionados y talleres al interior de nuestro equipo de trabajo.

\section{Análisis de posters producidos por estudiantes de la escuela secundariaiii (período 2017-2019)}

La producción de posters al finalizar el desarrollo de los talleres de intercambio entre estudiantes de las diferentes escuelas participantes resultó un modo de comunicar, abstraer y simbolizar las discusiones e ideas comunes al interior de cada grupo. 
El primer documento (poster) analizado, comienza diciendo: "Una experiencia significativa de aprendizaje en la escuela secundaria es..." y, a partir de ello, enumeran aspectos relacionados con la enseñanza de contenidos curriculares, con aspectos vinculares entre estudiantes y los/as docentes y aspectos ligados a los cambios personales/subjetivos que les posibilitaron las experiencias.

Los primeros aluden al tratamiento de temas curriculares vinculados con sus intereses y problemáticas, a clases dinámicas, activas, donde los/as jóvenes tengan protagonismo, con un/a profesor/a compenetrado/a en su oficio de enseñante que los empodere en el sentido de "pensar por uno mismo". Los segundos hacen referencia a procesos de socialización basados en el respeto y la incidencia de dichas experiencias en su subjetividad expresados en términos de "definir nuestro futuro o guiarnos" como expresan en la frase de cierre.

En el segundo poster, observamos la identificación de experiencias significativas de aprendizajes cuando estas promueven la potencialidad del desarrollo personal y una propia valoración de su presencia y participación, al mismo tiempo que cultiva actitudes favorables para la vida en sociedad, para la construcción de relaciones sociales sólidas y respetuosas de los/as otros/as y sus opiniones. Parece, entonces, que el tratamiento de un contenido se valora cuando ofrece la oportunidad de producir un pensar sobre las relaciones entre nociones, conceptos y conocimientos que posibilitan la comprensión/explicación de un fragmento de la realidad en y desde la que, docentes y estudiantes, interactúan.

Finalizan con la frase que dice: "la mente es como un paracaídas, no sirve si no se abre..."; "Un profe abre tu mente, te da una mano" (y dibujan una mano abierta).

La utilización de dibujos y frases elaboradas por ellos/as mismos/as nos indica una redimensión de los aspectos simbólicos que están siendo capaces de producir para definir al proceso educativo y donde, a través de esa simbolización, están expresando también una valoración de la tarea docente. Advertimos que, en esa valoración, encontramos rasgos que diferencian al docente modélico de otra época, del/de la docente próximo/a actual con el/la que, en oportunidades, pueden, inclusive, intercambiar el rol (respecto de la apropiación de contenidos curriculares, por ejemplo).

En el tercer poster, nuevamente, las estrategias de enseñanza son señaladas como aspectos relevantes al momento de pensar en experiencias significativas de aprendizaje. También recurren a identificar ciertos rasgos del oficio docente -como la paciencia, dedicación, preocupación por cada sujeto y su aprendizaje- en el que destacan "ir más allá" del rol de profesor/a y ejercer autoridad.

La simbolización de su agradecimiento dibujando un corazón dedicado a los y las docentes, que reconocieron como facilitadores de aprendizajes significativos, muestra la dimensión del aspecto afectivo de la tarea educativa, como la trama en la que pueden producirse esos procesos señalados por ellos/as como relevantes.

No parece casual que esta demanda explícita de los y las estudiantes en torno de su mayor actividad y participación en el acto educativo comienza a desplegarse con fuerza en un 
contexto de prácticas discursivas fuertemente atravesadas por los enunciados jurídicos en perspectiva de derechos. Esta línea se entrama con la perspectiva constructivista de la psicología que enuncia al sujeto de aprendizaje en/desde su lugar de protagonismo. La existencia de algunos procesos educativos, que han sido llevados adelante por profesores que han logrado mayores logros de expresión práctica de estos enunciados, han potenciado el desarrollo en ellos/as de estas ideas y reclamos.

Los dos últimos posters producidos reiteran y ratifican las ideas expresadas por los grupos antecedentes, donde los aspectos inicialmente señalados son reclamados como condición ineludible en la significatividad de los aprendizajes escolares.

Las expresiones de los y las jóvenes estudiantes a la hora de pensar en la significatividad del aprendizaje escolar parecen remitir con fuerza a la necesidad de que la experiencia educativa se vea atravesada por un encuentro interpersonal centrado en el respeto, el vínculo efectivo, la dedicación, compromiso y motivación para formar parte de este. Condiciones del encuentro que, por otro lado, parecen poner en el centro la pregunta por los sentidos de educar, en tanto oportunidad de promover un encuentro pedagógico que habilite a la transformación de quienes participan en el. Un por qué y para qué nos encontramos allí, unos sentidos ligados al desarrollo intelectual, reflexivo y crítico significado en un "abrir las mentes/cabezas, pensar por sí mismos/as, desarrollar las propias posturas" puestos al servicio de proyectar el futuro y promover el desarrollo personal como ciudadano.

La producción de la experiencia educativa parece requerir, aquí, de una participación activa y comprometida tanto de docentes como de estudiantes. Requiere del despliegue de una escucha y mirada atenta de un/a otro/a que tiene algo para aportar en la producción de un espacio de que habilite la co-construcción del devenir escolar. Esto es, una invitación conjunta y compartida a promover el deseo de aprender, conocer y pensar los contenidos de enseñanza, motivo de la transmisión, como un acto político que trasciende lo escolar, para proyectarse en la vida personal y social de quienes lo transitan. Es desde esta co-construcción e implicancia mutua que los/as estudiantes identifican la necesidad de dar lugar a otras relaciones posibles en el vínculo intergeneracional que la escuela promueve.

Estaríamos, así, ante un encuentro pedagógico que se transforma, "que, rompiendo con la lógica de producción moderna, pone en relación un tipo de vínculo que, lejos de presentarse regulado de ante mano, tiene lugar en el devenir de una experiencia que logra potenciar el interjuego entre afinidad y diversidad" (Viscaino, 2020, p. 5) entre posiciones en torno del saber y las figuras de autoridad que desde allí se logran producir.

Ese encuentro se evidencia permeado por un proceso de humanización de la relación, del vínculo, que torna novedosa y a la vez comprometida a la experiencia que se les ofrece (tanto a docentes como a estudiantes). 


\section{Análisis de las entrevistas realizadas con estudiantes de la escuela secundaria en ASPO (período 2020)}

En esta indagación se buscó relevar las percepciones y posibles repercusiones de las experiencias de aprendizaje llevadas adelante en el contexto del ASPO durante el año 2020. En particular se buscó indagar acerca de las condiciones en las que se llevaron adelante dichas experiencias en cuanto a: disponibilidad de dispositivos y conectividad, modos de producirse las comunicaciones entre docentes y estudiantes y entre estudiantes, dinámicas de los encuentros e intercambios, modos de vivenciar y participar de la experiencia estableciendo nexos de comparación con la presencialidad y tipos de experiencias que identificaban como más significativas y por qué las pensaban de ese modo.

En relación con la disponibilidad de recursos tecnológicos y conectividad, relevamos que el total de los/as jóvenes contaron con los dispositivos tecnológicos para mantener el contacto con la escuela y realizar las actividades (mayoritariamente, teléfonos celulares con datos o conexión a internet en el hogar).

Respecto a las condiciones del hogar para realizar las tareas escolares, poco más del $50 \%$ dispone de un espacio físico apropiado (aislado del bullicio del hogar) para hacer las tareas escolares o tener clases sincrónicas. Expresan también que las familias se ocuparon de organizar parte de la dinámica cotidiana de modo tal de no interferir en las tareas escolares. Lo mismo sucede con el uso de dispositivos tecnológicos, ya que las familias privilegiaron el uso de estos (cuando eran compartidos), en los horarios escolares, para tal fin. Para las tareas grupales, hemos relevado que gran parte de las y los jóvenes preferían realizar los encuentros en horarios nocturnos cuando "había silencio en la casa" y "todos ya se habían ido a dormir".

Interpretamos, a partir de estas dos últimas expresiones, que la excusa de la tarea escolar favoreció el mantenimiento de espacios propios donde las y los jóvenes tuvieran encuentros, en período de ASPO más estricto, para realizar encuentros sociales sin la presencia de adultos.

Respecto de la dinámica de las clases, relatan que los/as docentes, en el inicio de la nopresencialidad, trasladaron sus clases habituales a las plataformas disponibles y que, paulatinamente, comenzaron a "ser más selectivos" en la propuesta de temas de enseñanza, tal como señala una joven. Explicó, luego, que profesores y profesoras se ajustaron a los núcleos prioritarios de contenidos que señaló el gobierno y buscaron dar a las clases una impronta problematizadora, buscando estrategias para que las y los estudiantes se involucraran más.

Respecto de esto último, el trabajo colaborativo fue uno de los aspectos que presentó un alto nivel de desconocimiento (60\%), en especial en el uso de herramientas digitales para tal fin. Aun reconociendo su importancia, más del $80 \%$ de las y los jóvenes manifiestan que preferían hacer los trabajos de manera individual. Algunos/as más preocupados/as por su trayectoria, decían asegurarse, así, de entregar los trabajos en tiempo y forma.

Con relación a la significatividad de las experiencias de aprendizaje en este contexto, del total de las 30 entrevistas, solo cinco jóvenes pudieron identificar alguna acción que, desde lo 
escolar, le produjo una huella, se inscribió en su experiencia. Los restantes veinticinco manifiestan que no hicieron, durante todo el año, nada significativo. Entre los argumentos que ellos/as presentan, señalan la gran cantidad de trabajos que tenían que realizar y entregar a sus profesores para mantener el ritmo y trayectoria esperada de las cursadas.

Aquí, la entrega de trabajos se constituía para ellos/as como una actividad que solo se producía para responder al pedido de un/a docente sin demanda de implicación personal en su resolución. Referencias que suelen estar acompañadas con expresiones del tipo "yo no aprendí nada 'serio'" o no haber aprendido un "tema específico" o señalar la diferencia de realizar un trabajo solo para entregar en el que "no tomas conciencia de cada palabra que pones" a diferencia de lo que sucede cuando es algo pensado, que atraviesa personalmente.

Para quienes, por el contrario, encontraron algunas experiencias de significatividad en este proceso, estas residían en el requerimiento de algún tipo de reflexión, análisis e involucramiento personal que implicó algún tipo de repercusión especial en su desarrollo

Tal como plantea una estudiante de una escuela privada, confesional, ubicada en una localidad próxima a la ciudad cabecera de partido, identifica una actividad (ensayo) que realizó en la materia Filosofía para ser presentado en las Olimpíadas Argentinas de Filosofía, en la que se sintió más "tocada en lo personal" en tanto fue un trabajo que la llevó a "cambiar la forma de pensar". 0, como refiere otra estudiante de la misma escuela, ante la descripción de una actividad que le permitió conectarse con otras instituciones y conocer "un montón de gente" y llevar adelante la creación de un video sobre las noticias y las fakenews que le implicó un "empeño" especial en su "diseño".

Para otra estudiante de una escuela confesional de la localidad, la experiencia de diseñar un video también fue recuperada como un tipo de actividad que la ha motivado y resultó entretenida, ya que le permitió competir con propuestas de otras instituciones o entre grupos escolares y en los que se vieron implicadas más de una asignatura y docentes.

También encontramos una expresión de "disfrute" expresada por un estudiante de sexto año de la escuela pública tradicional, quien relata una experiencia vivida en la materia Historia, en la que debían analizar canciones de rock nacional. Esta actividad le permitió "despedazar" estas descubriendo, en ese análisis, "todo lo que hay detrás de cada canción" y la atención e impacto que este trabajo le produjo en el cambio de perspectiva y el uso de otro lenguaje que pudo trasladar a su actividad como compositor. Otra estudiante, de la escuela secundaria rural, en la misma línea, remite descubrir el gusto por una materia como Psicología cuando le permite trabajar con contenidos que la involucran en su etapa adolescente y en "cosas que pasan en la vida" ayudándola a "entender cosas para la vida" cuando se le solicita hacer un trabajo reflexivo.

De esta manera, la reflexión que diferencia aprendizaje y entrega de trabajos parece involucrar de forma sustancial al tipo de propuesta docente, la forma de enseñar y evaluar los aprendizajes que un/a docente lleva adelante y cuándo se considera que un contenido ha sido aprendido. Así, ellos/as interpretan la insignificancia de la entrega de trabajos, del cumplimiento de las demandas docentes por sobre los tiempos, las experiencias, los sentidos y sentimientos que 
logran promover en ellos/as aprendizaje con sentido personal y social, dando visibilidad a la distancia o vacío que suele existir entre dos procesos que deberían estar relacionados íntimamente, intrínsecamente: enseñar y aprender.

\section{La significatividad en su dimensión subjetiva y sociocultural}

Es posible observar, del material descripto hasta aquí, la presencia de una significativa coincidencia de sentidos producidos por estudiantes de diferentes instituciones educativas y contextos de producción de la experiencia escolar, en torno de las condiciones que promueven o inhiben la significatividad de esta. Estas condiciones se centran con fuerza en la presencia o ausencia de un Ilamado, una invitación a participar, implicarse y pensar en torno de un contenido escolar puesto en diálogo con la vida social y sus experiencias vitales en otros ámbitos y contextos culturales por fuera de la institución escolar.

A partir del análisis de las expresiones presentadas, encontramos que la significatividad estará dada aquí por la valoración particular que realizan los sujetos de aquellas experiencias de aprendizaje escolar que tienen la capacidad de "ofrecerles" o "dejarles" algo importante, una marca, un registro que, recuperado a la distancia, significa o adquiere significado por su repercusión actual. Una repercusión que trasciende el tiempo y espacio escolar, recuperándolo y resignificándolo a posteriori.

De esta manera, y recuperando algunos pensamientos de Frigerio (2004), podríamos identificar en los y las estudiantes una demanda de trabajo pedagógico que recupere "un más allá" del contenido escolar objeto de la transmisión. Un más allá que se ubica en la relación y se produce en el acto mismo de la transferencia producida en y desde un lazo, un vínculo que define el encuentro intergeneracional y lo humaniza. Un amor de transferencia, nos dice la autora, que, lejos de ser obstáculo, se convierte en soporte de la transmisión y en un juego de desplazamientos, circule y alcance tanto a los objetos y como a los sujetos de dicha transmisión, produciendo un plus, un trabajo (Arbeit) que, excediendo el contenido de lo que se transmite, modifica a los sujetos que participan de la experiencia.

Esa repercusión subjetiva y social del encuentro pedagógico logra desplegarse toda vez que el mismo posibilitó la producción de vínculos de respeto, escucha, confianza y reconocimiento entre los participantes de la actividad y desde el que ha sido posible la habilitación de un pensar y actuar colectivamente en la situación. Es desde este tipo de intercambio que los objetos de la transmisión escolar parecen haber producido algo de ese plus, al que refiere Frigerio (2004), que promueve la comprensión, "aplicación" o "transferencia" del contenido escolar, tal como lo expresan a otros campos de saber o de prácticas sociales de su interés. Un sentido social y cultura/del encuentro pedagógico que se ve atravesado por la gratificación y emoción que puede producir la percepción de dominio y potencia de sí y del entorno. Es en este entramado significante que los relatos de los sujetos entrevistados presentan como sello distintivo la implicación personal y la participación social. Los sujetos anteponen la significatividad como un proceso subjetivo y social de la experiencia de aprendizaje; o quizá aún 
más: la consideran una variable sustantiva, indispensable, para que existan la experiencia y el aprendizaje escolar.

Finalmente, y recuperando el desarrollo presentado en el segundo apartado de este escrito, podríamos pensar que el acercamiento a los y las jóvenes estudiantes del nivel secundario nos estaría permitiendo recuperar, resignificar o complejizar algunos de los aportes teóricos, antes mencionados, y que nos aporta la psicología del siglo XX.

En particular, quisiéramos señalar dos aspectos claves de nuestra contribución a la comprensión de la significatividad de las experiencias de aprendizaje que hemos relevado en las aulas contemporáneas de nivel secundario. El primero corresponde a un análisis en perspectiva psicológica y, el segundo, a una perspectiva sociológica, aunque ambos se encuentren entrelazados.

Desde las voces de los y las entrevistadas, podemos identificar que la mirada de la comprensión y significatividad de un contenido escolar estaría dada por la implicancia de un diálogo permanente entre el pensar, reflexionar, vincular lo nuevo con lo conocido, en un trabajo de revisión de sí mismo y su actuación en su mundo circundante. Así, una referencia al contenido de enseñanza y sus posibilidades de producir reorganización conceptual, desde una perspectiva cognitiva, como la que nos ofrece Ausubel, se vería aquí resignificada por los sentidos sociales y culturales que los mismos tienen para su vida, su contexto y grupo de referencia. Parece relevante el modo en que los/as entrevistadas refieren a los cambios de perspectiva, mirada, comprensión de sí y de su actuación en la vida social presente y futura que pudieron representar algunas experiencias significativas del ámbito escolar. En términos de la perspectiva sociocultural de Bruner y Vygotski, podríamos afirmar que la actividad social y cultural que se promueve en y desde el dispositivo escolar es representada como experiencia significativa o cargada de significatividad cuando ha permitido cambiar/ampliar/reorganizar su visión/comprensión de un proceso social, puesto a trabajar con otros/as en un ámbito público como el que la escuela posibilita.

Los estudios realizados nos posibilitan sostener la idea de que la función educadora centrada en la transmisión de contenidos curriculares que realizara la escuela secundaria en épocas de secuencia institucional, hoy, está siendo desbordada y la escuela está llamada a realizar una supuesta contención de los/as jóvenes, que encuentran pocos espacios donde experimentar, desde allí, lo propio de su experiencia vital. La pandemia y el aislamiento social que se impuso en nuestro país dejó al descubierto la "añoranza" de la descalificada escuela física en tanto espacio de encuentro. Aunque la mudanza hacia la virtualidad no pareció tener un impacto en la mejora de las experiencias de aprendizaje, sí podríamos afirmar que nos ha dejado indicios valiosos para su revisión.

\section{Conclusiones}

A la luz de las ideas en proceso que se corresponden con esta etapa de la educación que se conoce como "cambio educativo" (Hargreaves, 2003), se articulan y recomponen viejas ideas para nuevos problemas y hacia ellos debieran estar mirando las instituciones y los sujetos. 
Al inicio del presente escrito, hacíamos referencia a los profundos cambios sociales, culturales, políticos y tecnológicos que atraviesan las sociedades contemporáneas y, con ellas, las instituciones y los sujetos que las habitan. Los procesos de investigación emprendidos en los últimos diez años nos han permitido redimensionar dichas tensiones en el contexto de ampliación del nivel de educación secundaria. Y, desde allí, relevar las tensiones producidas al interior de las dinámicas escolares para conciliar masificación e inclusión y, en consecuencia, producir una revisión de sentidos y prácticas que le dieron forma en sus inicios.

Las voces de quienes transitan la escuela secundaria en el tiempo actual (Errobidart, 2014, 2016) o quienes han dejado de hacerlo (Viscaíno, 2018) ponen en jaque los sentidos, prácticas y dinámicas que dieron forma a la escuela moderna en general y los sentidos identitarios del nivel secundario en particular. Sus voces cobran especial fuerza en el nuevo entramado intergeneracional y el contexto de cambio y conmoción que, como sociedad, transitamos en estos tiempos. Sus voces nos remiten a un lugar desde el cual visibilizar la propia actuación personal como parte de una construcción sociocultural que hace a la vida en común.

El contrato social que la escuela de la modernidad establece con la sociedad de su tiempo sufre la dilución de tiempos contemporáneos. Y, podríamos afirmar, que es de escucha atenta lo que las nuevas generaciones nos dan a ver y pensar en relación con el qué, cómo y por qué del trabajo escolar. Una revisión que, en tanto educadores, nos reedita el sentido político y subjetivo del acto de educar.

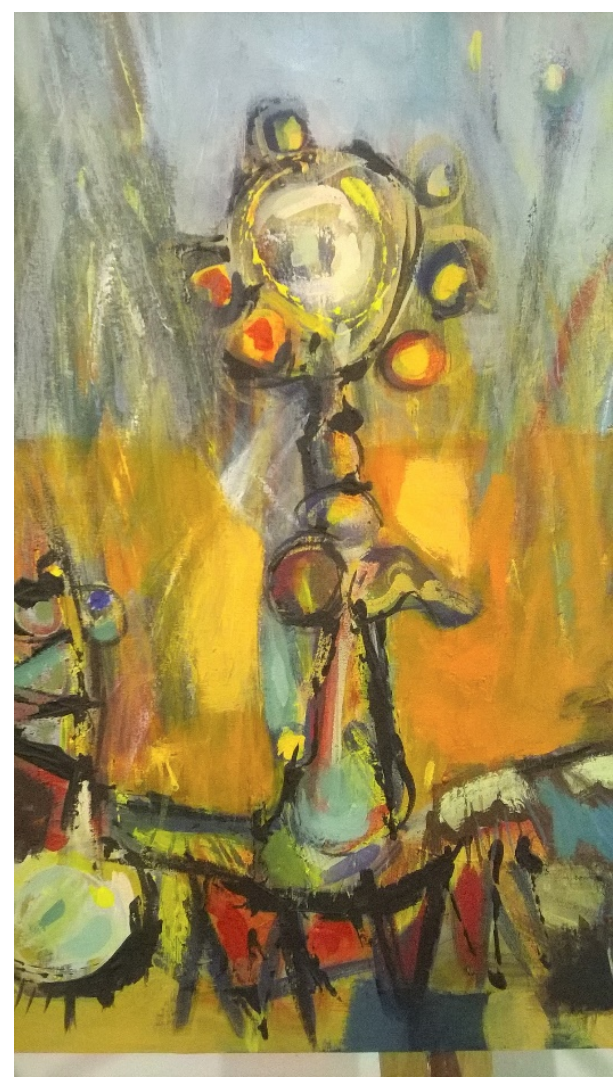

\section{ST, acrílico. Noemí Fiscella}




\section{Bibliografía}

Ausubel, D., Novak, J. y Hanesian, H. (1991). Psicología Educativa, un punto de vista cognoscitivo. Editorial Trillas.

Ausubel, D. P. (1973). La educación y la estructura del conocimiento. Investigaciones sobre el proceso de aprendizaje y la naturaleza de las disciplinas que integran el currículum. El Ateneo.

Bruner, J. (1998). Realidad mental y mundos posibles. Los actos de imaginación que dan sentido a la experiencia. Gedisa.

Bruner, J. (1991). Actos de significado. Más allá de la revolución cognitiva. Editorial Alianza.

Chapato, M. E. y Errobidart, A. (Comp.). (2011). Historias, actores e instituciones. Estudios sobre prácticas educativas en tiempo de cambios y turbulencias. Miño y Dávila.

Dubet, F. (2010). El declive de la institución. Profesiones, sujetos e individuos en la modernidad. Cedisa. Dubet, F. (2011). Repensar la justicia social. Contra el mito de la igualdad de oportunidades. Siglo XXI. Errobidart, A. (2014). Con las mejores intenciones. Estudio sobre las prácticas políticas de los jóvenes estudiantes en la actual escuela secundaria obligatoria, en un contexto de fragmentación social[Tesis doctoral]. http://hdl.handle.net/10915/44513 Errobidart, A. (directora). (2016). Trazos de escuela. Un abordaje etnográfico en la educación secundaria. Miño y Dávila.

Errobidart, A. (2021). Perspectiva socioantropológica para investigar los sentidos de la educación que se construyen bajo el mandato de inclusión social. En A. Errobidart y C. Casenave (Comp.), Inclusión con calidad de los aprendizajes en la escuela secundaria. Sobre mandatos, controversias y continuidades. Ed. UNICEN. https://www.soc.unicen.edu.ar/images/editorial/ebooks/errobidart2021.pdf Frigerio, A. (2004). Los sentidos del verbo educar. https://docs.google.com/viewer?a=v\&pid=sites\&srcid=ZGVmYXVsdGRvbWFpbnxpc2ZkcGVkYWdvZZIhMjAxN HxneDo3Nm/hNjBkMjcyMjJhOTII

Gimeno Sacristán, J. (2013). En busca del sentido de la educación. Morata.

Giroux, H. (1992). Teoría y resistencia en educación. Paidós.

Grimson, A. y Tenti Fanfani, E. (2014). Mitomanías de la Educación argentina. Crítica de las frases hechas, las medias verdades y las soluciones mágicas. Siglo XXI.

Hargreaves, A. (Comp.). (2003). Replantear el cambio educativo. Un enfoque renovador. Amorroutu. Lewkowicz, I. (2004). Escuela y ciudadanía. En A. Corea e I. Lewkowicz, Pedagogía del aburrido. Escuelas destituidas, familias perplejas. Paidós.

Pérez Gémez, A. (2017). Pedagogías para tiempos de perplejidad. Homo Sapiens.

Tenti Fanfani, E. (2007). La escuela y la cuestión social. Ensayos de sociología de la educación. Siglo XXI. Tiramonti, G. (2011). Variaciones sobre la forma escolar. Límites y posibilidades de la escuela media. FLACSO. Homo Sapiens.

Vygotski, L. S. (2000). El desarrollo de los procesos psicológicos superiores. Editorial Crítica/Grijalbo. Viscaino, A. M. (2018). La filiación simbólica en adolescentes que no asisten a la "nueva escuela secundaria". La promesa incumplida de ser alguien en la experiencia pedagógica política de producir lo común [Tesis doctoral]. Facultad de Ciencias de la Educación, Universidad Nacional de Entre Ríos. http://biblio.fcedu.uner.edu.ar/listados/TESIS_DOCTORADO_EN_EDUCACION. Viscaino, A. M. (2020). El encuentro pedagógico En busca de mediaciones que potencien presencias. Revista Te\&Te, (28). http://teyet-revista.info.unlp.edu.ar/ 
"Vygotski pronuncia una conferencia titulada "La conciencia como objeto de la psicología de la conducta" en el Segundo Congreso Psiconeurológico de Moscú, en 1924. Tanto aquí como en publicaciones posteriores, desarrolla una fuerte crítica a la dificultad de la Psicología de la época para encontrar una base sólida que sustente una teoría unificada de los procesos psicológicos humanos en toda su complejidad.

ii Proceso de internalización que queda ejemplificado en uno de sus escritos a través de una hermosa escena que muestra a un niño pequeño que levanta sus manos en busca de un objeto alejado de sí y, a partir de la reacción e intervención de su madre, que interpreta el movimiento del niño como un pedido, el acto de asir se transforma en el acto de señalar (p. 93). La acción de tomar, en tanto acto dirigido al objeto externo, se modifica sustancialmente con la reacción de otra persona en un gesto, ahora, dirigido a otros. El significado y sus funciones se van reconstruyendo, en el interior del niño, a través de un proceso intrapersonal que nace de la actividad externa y el encuentro intersubjetivo en el mundo social.

iii Esta actividad de cierre de los talleres se realizó con 250 estudiantes de sexto año de las escuelas vinculadas a la investigación. En el encuentro presencial, cada pequeño grupo aportaba una idea para la producción de un poster/afiche y elaboraban una idea común de cierre. Cada grupo, coordinado por dos investigadoras, integraba a jóvenes de diferentes escuelas. 